\title{
In situ X-ray Diffraction of Lead Zirconate Titanate PiezoMEMS Cantilever During Actuation
}

Giovanni Esteves ${ }^{\mathrm{a}}$, Chris M. Fancher ${ }^{\mathrm{a}}$, Margeaux Wallace ${ }^{\mathrm{b}}$, Raegan Johnson-Wilke ${ }^{\mathrm{b}}$, Rudeger H. T. Wilke $^{\mathrm{b}}$, Susan Trolier-McKinstry ${ }^{\mathrm{b}}$, Ronald G. Polcawich ${ }^{\mathrm{c}}$, and Jacob L. Jones ${ }^{\mathrm{a}}$

North Carolina State University, Department of Materials Science and Engineering, Raleigh, NC

b. The Pennsylvania State University, Department of Materials Science and Engineering, University Park, PA

c. United States Army Research Laboratory, Adelphi, MD

\begin{abstract}
Synchrotron X-ray diffraction (XRD) was used to probe the electric-field-induced response of $500 \mathrm{~nm}$ lead zirconate titanate $(52 / 48, \mathrm{Zr} / \mathrm{Ti})$ (PZT) based piezoelectric microelectromechanical system (piezoMEMS) device. $90^{\circ}$ ferroelectric/ferroelastic domain reorientation was observed in a cantilever comprised of a $500 \mathrm{~nm}$ thick PZT film on a $3 \mu \mathrm{m}$ thick elastic layer composite of $\mathrm{SiO}_{2}$ and $\mathrm{Si}_{3} \mathrm{~N}_{4}$. Diffraction data from sectors both parallel- and perpendicular-to-field sectors showed the presence of ferroelastic texture, which is typically seen in in situ electric field diffraction studies of bulk tetragonal perovskite ferroelectrics. The fraction of domains reoriented into the field direction was quantified through the intensity changes of the 002 and 200 diffraction profiles. The maximum induced volume fraction calculated from the results was $20 \%$, which is comparable to values seen in previous bulk and thin film ferroelectric diffraction studies. The novelty of the present work is that a fully released ferroelectric thin film device of micron scale dimensions (down to $60,000 \mu \mathrm{m}^{3}$ ) was interrogated in situ with applied electric field using synchrotron XRD. Furthermore, the experiment demonstrates that $90^{\circ}$ ferroelectric/ferroelastic domain reorientation can be characterized in samples of such small dimensions.
\end{abstract}




\section{Introduction}

Piezoelectric microelectromechanical systems (piezoMEMS) are MEMS devices which utilize piezoelectric films to convert between electrical and mechanical energies. Lead zirconate titanate $\left(\mathrm{PbZr}_{\mathrm{x}} \mathrm{Ti}_{1-\mathrm{x}} \mathrm{O}_{3}\right)(\mathrm{PZT}(\mathrm{Zr} / \mathrm{Ti}))$ thin films with a composition close to the morphotropic phase boundary (MPB), $x=0.52$, are widely used in piezoMEMS due to their high piezoelectric response.[1] The high piezoelectric constants of high performance PZT thin films enables low voltage mm-scale robotics.[2-4] Miniaturizing robotics could provide benefits in defense applications, including higher levels of stealth entry and access to locations that would otherwise be un-achievable. Depending on the configuration of the PZT film, piezoMEMS actuators are capable of generating lateral, vertical, and torsional motion. This allows PZT films to be utilized as motion generators for insect-like microrobotics.

The electromechanical response of ferroelectric materials is influenced by two main contributions; (1) the intrinsic piezoelectric effect, and (2) extrinsic contributions, predominantly from domain wall motion. In bulk PZT ceramics, domain wall motion contributes a large fraction of the overall piezoelectric response of the material.[5] In general, there are two types of domain wall motion, $180^{\circ}$ and non- $180^{\circ}$. The domain wall contributions to the piezoelectric response is typically dominated by non- $180^{\circ}$ domain wall motion,[5-7] due to the field-induced ferroelectric/ferroelastic distortions that accompany motion of these walls. $180^{\circ}$ domain walls can also add to the piezoelectric response, as seen in work done on PZT thin films under high ac fields.[8] Ultimately, non- $180^{\circ}$ domain reorientation can be tracked as a function of electric field by diffraction methods, including XRD.

The application of electric field during diffraction measurements can provide useful information regarding the electromechanical response of the ferroelectric sample.[9-12] The 
electric field induced lattice strain of a ferroelectric material can be calculated from peak shifts. In diffraction measurements, intensity exchanges between degenerate reflections, e.g. 002 and 200 for tetragonal ferroelectrics, can yield quantitative domain reorientation information.[13-18] Meanwhile, disappearance and appearance of peaks for on and off-field measurements, can help identify electric-field-induced phase changes.[14,19]

In thin films, the extrinsic contribution can be affected by crystallographic texture, grain size, and residual stress.[3,20-24] The crystallographic texture of the ferroelectric film controls the amount of domain reorientation, with $\{001\}$ films showing the highest amount of non- $180^{\circ}$ domain reorientation in tetragonal perovskites.[25,26] Small grain sizes are known to lead to increased pinning of domain walls, $[24,27]$ and residual stresses from the growth process within the ferroelectric film can reduce domain wall mobility.[25,28] It is worth noting that these residual stresses are a product of all of the previously mentioned factors and can also change as a function of film thickness, ultimately affecting the dielectric response of the film.[29] For example, after processing ferroelectric films grown on silicon substrates, the film is subjected to tensile stress, after cooling, due to the thermal expansion mismatch between the film and substrate.[30] The tensile stress yields a preferred polarization in the plane of the film, and increases the energy barrier to reorient the polarization of domains out-of-plane.[31]

Altering the mechanical boundary constraints, by substrate removal and/or introduction of porosity to the ferroelectric film influences the film's domain reorientation response. For example, Wallace et al.,[32] used synchrotron X-ray diffraction (XRD) on $\{100\}$ textured PZT films to show that the removal of $\sim 75 \%$ of the underlying substrate led to a six times increase in the amount of domain reorientation. The partial removal of the substrate allowed for the in-plane stress to be partially relieved, thus, lowering the energy barrier for inducing domain 
reorientation. A similar study comparing porous and dense as-processed $\{100\}$ PZT films found that porous films achieved higher domain reorientation values.[33] This increase in domain reorientation was attributed to a reduction in domain wall pinning as a result of lowering the film stiffness due to porosity within the film, lowering the average stress state, and/or changing field concentrations. Thus, the mechanical boundary constraints can modulate the domain reorientation response in ferroelectric thin films.

Micro-fabricated PZT cantilevers have dramatically different boundary constraints than as-processed films. PZT cantilevers have been studied both through electrical property measurements and with XRD. Morioka et al.,[34] has studied the electric-field structural response of $1.1 \mu \mathrm{m}$ thick PZT (44/56) using a lab source diffractometer and was able to confirm $90^{\circ}$ domain reorientation. The composition of this PZT film lies on the tetragonal side of the MPB for the PZT binary system, which in combination with a thick film allows a lab source diffractometer to adequately probe the materials response. Similarly, Morioka et al.,[35] conducted a similar study on a microcantilever utilizing a $1.1 \mu \mathrm{m}$ thick PZT (52/48) in which no $90^{\circ}$ domain reorientation was observed. Materials using PZT compositions close to the MPB become more challenging to probe with XRD, especially when utilizing a lab source. Many advances have been made in the field of ferroelectrics due to the availability of synchrotron sources, which can enable improved crystal structure determination and give insight into the average response of the material.

In the present work, the electromechanical response of a functional piezoMEMS device utilizing $500 \mathrm{~nm}$ thick PZT (52/48), was probed in situ using synchrotron XRD. The film measured in the present study is thinner than those investigated by Morioka et al.,[34,35], and the use of a synchrotron source offers enhanced resolution compared to a lab source 
diffractometer. Earlier measurements done on ferroelectric thin film capacitors have been conducted on electrode areas of $15 \mathrm{~mm}^{2},[32,33,36]$ this study explores increasingly lower limits by studying an electrode area of $\sim 0.118 \mathrm{~mm}^{2}$ on a piezoMEMS device. From the XRD data the domain reorientation response was calculated through the integrated intensity of degenerate reflections, i.e. 002 and 200 for tetragonal ferroelectrics. Quantified domain reorientation values were on the same order of domain reorientation values calculated for previous bulk ferroelectrics studies. This study highlights the capability of using XRD to characterize ferroelectric films with small area capacitors.

\section{Experimental Procedure}

The fabricated piezoMEMS device utilized a $\mathrm{Pb}\left(\mathrm{Zr}_{0.52} \mathrm{Ti}_{0.48}\right) \mathrm{O}_{3}$ thin films synthesized using chemical solution deposition at the US Army Research Laboratory. Details of the sample synthesis, including the layer stacking is described in Ref. [37]. In short, the composite actuators are comprised of $3 \mu \mathrm{m}$ thick elastic layer comprised of a multilayer of $\mathrm{SiO}_{2}$ and $\mathrm{Si}_{3} \mathrm{~N}_{4}$ thin film, a 35nm thick $\mathrm{TiO}_{2}$ layer, a 100nm Pt bottom electrode, a 500nm PZT (52/48) actuator layer, and a

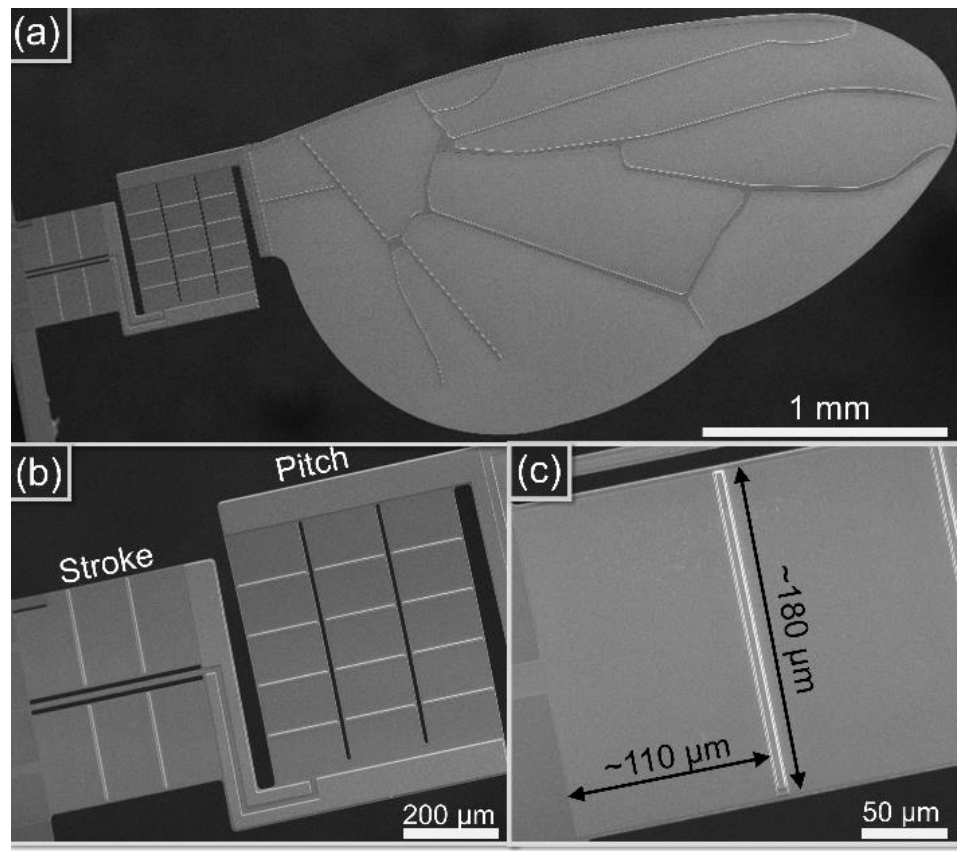

Figure 1. SEM images of (a) Fabricated MEMS device, (b) stroke and pitch component that utilize PZT, and (c) zoomed in portion of the stroke displaying approximate dimensions of electrode area. 
$100 \mathrm{~nm}$ Pt top electrode. The PZT film was targeted for a thickness of $500 \mathrm{~nm}$ with a $\{100\}$ crystallographic texture. Since the piezoMEMS mm-scale wing closely resembles a cantilever geometry, the term PZT cantilever will be used interchangeably to refer to the piezoMEMS device. Fig. 1 (a) shows an SEM image of the fully fabricated piezoMEMS device. The actuation motion comes from two PZT films oriented orthogonal to one another, producing two types of motions that can be individually actuated, stroke and pitch, shown on Fig 1 (b).

In this experiment, only the stroke portion of the cantilever was actuated, irradiated, measured, and quantified in order to reduce the signal of gold, platinum, and chromium on the pitch portion of the cantilever. The volume irradiated of electrically-active PZT was $\sim 60,000$ $\mu \mathrm{m}^{3}$, which is approximately equivalent to irradiating a human hair with 60 microns in diameter and 21 microns in length. SEM images of the plan view of the stroke and the pitch are shown in Fig. 1.

An in situ electric field dependent diffraction experiment was carried out at beamline 11ID-C of the Advanced Photon Source (APS) at Argonne National Laboratory using a Perkin Elmer detector placed approximately two meters away from the sample. This is a similar setup which has been reported previously in references $[32,33,36]$. Using a constant wavelength of $\lambda=0.10801 \AA$, a beam $350 \mu \mathrm{m}$ tall by $400 \mu \mathrm{m}$ wide was used to optimize the diffraction signal of the stroke portion of the PZT cantilever. This beam remained on the probed actuator during electric-field-driven-motion of the cantilever. A standard sample, cerium dioxide $\left(\mathrm{CeO}_{2}\right)$, was used to calibrate the sample-to-detector distance, beam center, and detector orthogonality. Voltage was applied using a Keithley $2410 \mathrm{C} 1100 \mathrm{~V}$ source meter. The applied voltage was incrementally increased to a maximum of $20 \mathrm{~V}$ in $5 \mathrm{~V}$ increments, then decreased down to $0 \mathrm{~V}$. 
The XRD patterns were measured for 5 minutes at each voltage step to obtain data with reasonable statistics because the irradiated volume was small.
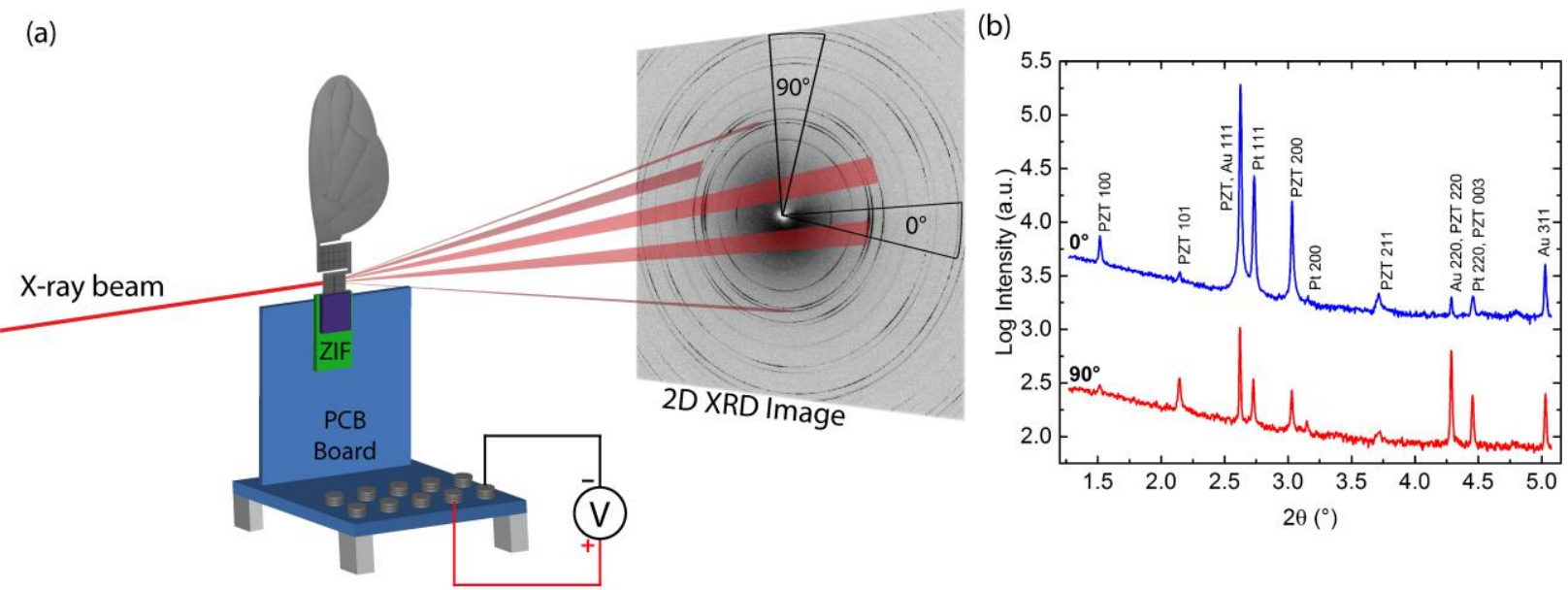

Figure 2 a) Experimental setup used for in situ diffraction experiment at 11-ID-C, and b) sectors chosen for integration on $2 \mathrm{D}$ diffraction image, $0^{\circ}$ sector describes the parallel-to-field direction and $90^{\circ}$ sector describes the perpendicular-to-field direction.

A special stage was designed for this experiment to apply electric fields to the PZT cantilever. A 24-pin zero insertion force (ZIF) socket was mounted on a printed circuit board (PCB) with wires soldered from the ZIF socket to another PCB board that allowed application of an electric field via alligator clips. To attach the special PCB stage onto the stage at 11-ID-C, an additional attachment stage was fabricated. This attachment stage allowed for minimal movement of the PCB stage when removing and attaching the alligator clips. A full setup is shown in Fig. 2 (a). The PZT cantilever was mounted with the stroke portion of the cantilever aligned parallel to the X-ray beam; this allowed the crystallographic texture of the PZT thin film to be measured during acquisition.

The Fit $2 D$ program was used to reduce the 2D diffraction images, obtained from synchrotron measurements, in order to perform further analysis.[38] The 2D diffraction patterns were reduced to $1 \mathrm{D}$ plots of $2 \theta$ versus intensity for sectors that represent scattering vectors that are parallel $\left(0^{\circ}\right)$ and perpendicular $\left(90^{\circ}\right)$ to the applied electric field direction. The integration 
range used in Fit $2 D$ for each sector was $20^{\circ}$ in the azimuthal direction of the $2 \mathrm{D}$ image. Figure 2 (b) illustrates the sections integrated on the $2 \mathrm{D}$ image that were used for data analysis and discussion. The importance of both parallel- and perpendicular-to-field sectors is the orientation of the lattice planes probed relative to the applied field. For example, the parallel-to-field sector probes lattice planes that are oriented parallel to the surface of the film, i.e. oriented perpendicular-to-electric-field direction; this yields information about the out-of-plane response of the film. The perpendicular-to-field sector probes lattice planes that are perpendicular to the surface of the film, i.e. oriented parallel to the electric field direction, and yields information about the in-plane response of the film.

\section{Discussion}

Figure 3 (a) shows evidence of domain reorientation between the $0 \mathrm{~V}$ and $20 \mathrm{~V}$ diffraction patterns of the 002 and 200 diffraction peaks. The development of the 002 reflection upon application of electric field shows direct evidence of $a$-domains reorienting to $c$-domains. When an electric field is applied, the fraction of $c$-domains increases due to $90^{\circ}$ ferroelectric/ferroelastic domain reorientation. The behavior is seen in diffraction patterns by an increase in intensity of the 002 in parallel-to-field direction. The absences of the 002 reflection, for the initial diffraction pattern at $0 \mathrm{~V}$, can be explained as the initial volume of $c$-domains being too small to be detected by X-rays, as shown in Fig. 3 (a). When an electric field is applied, some $a$-domains switch to $c$-domains, thus, increasing the volume of $c$-domains. The resulting increase in volume of $c$-domains is significant enough to be distinguished by $\mathrm{X}$-rays, leading to the development the 002 peak. These results indicate that the amount of domain reorientation is substantial, which has been seen in bulk PZT MPB compositions, [39] but not on PZT films that are less than $1 \mu \mathrm{m}$ due to the available XRD instrumental resolution.[35] 


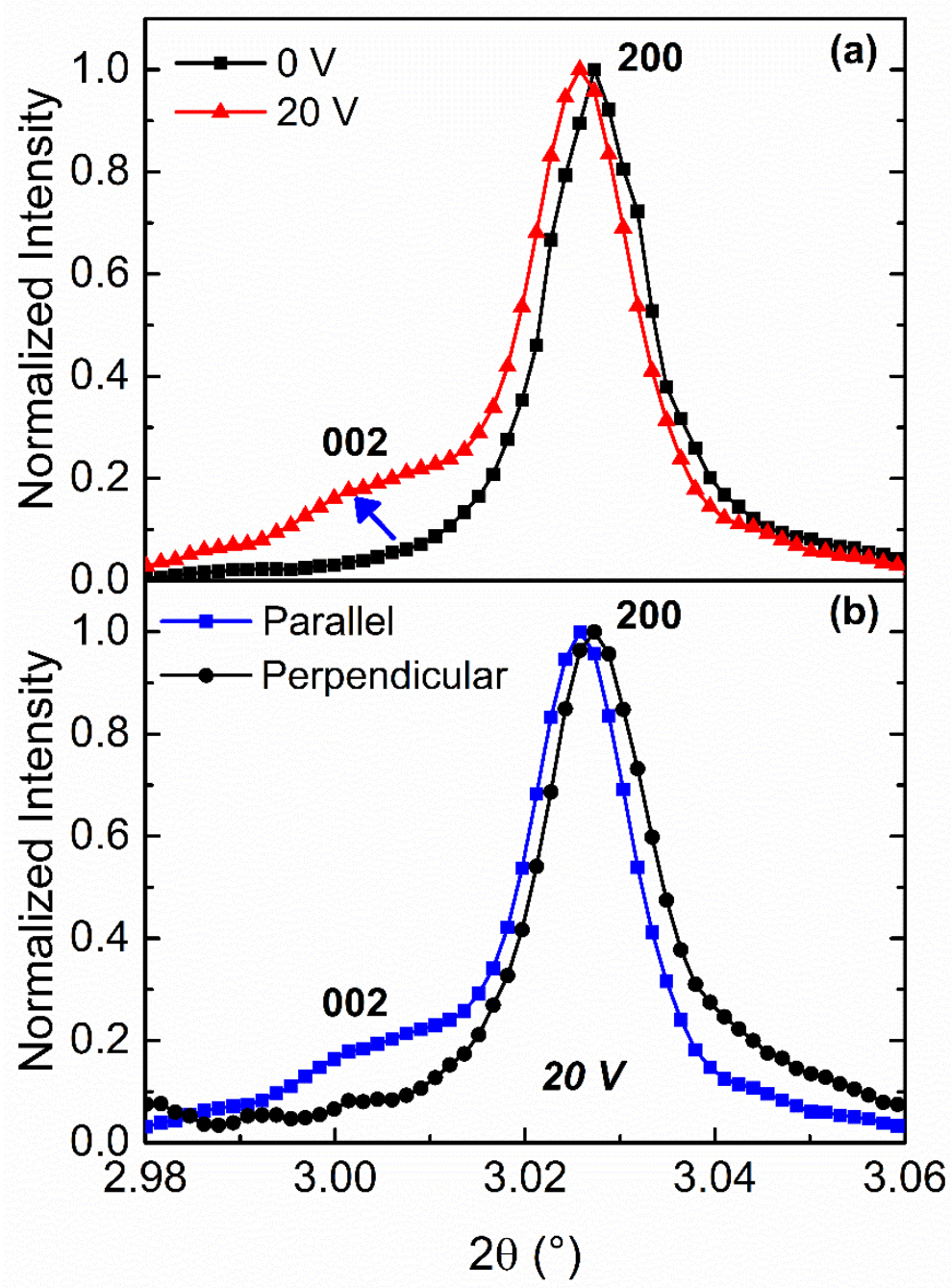

Figure 3 1D diffraction patterns of 002/200 reflections, (a) at $0 \mathrm{~V}$ and 20 $\mathrm{V}$, and (b) comparing both diffraction signal from scattering vectors that are parallel and perpendicular to electric field.

In Fig. 3 (b) the absence of a PZT 002 peak at perpendicular-to-field direction provides further evidence of the existence of $90^{\circ}$ domain reorientation. When domain reorientation occurs within a ferroelectric material, its effect can be seen in the diffraction pattern as a preferred distribution of intensities, referred to as ferroelastic texture. This ferroelastic texture is most prominent in the parallel- and perpendicular-to-field directions. Since intensity is conserved when comparing diffraction signal from various scattering vectors, an increase in the 002 reflection in the parallel-to-field direction leads to a decrease in the perpendicular direction, as 
seen in many bulk ferroelectric studies.[14,40] Based on the present results, this suggests that the majority of the $c$-oriented domains have switched to the out-of-plane direction, and thus, are measured in the parallel-to-field sector at $20 \mathrm{~V}$.

MPB bulk PZT has been known to undergo electric-field-induced phase transitions.[19,41-43] However, the films explored in the present work are assumed to be single phase tetragonal and do not exhibit an electric-field-induced phase transition. Due to the low diffraction signal from the PZT cantilever and small irradiated volume, it is difficult to obtain intensity from $00 \mathrm{l}$ reflections at $0 \mathrm{~V}$, which would help in further distinguishing the initial phase of the film. If a phase transition was occurring this means the structure of the PZT film, at $0 \mathrm{~V}$, starts as a rhombohedral structure and upon electric field application a phase transition occurs from rhombohedral to tetragonal. This scenario seems less likely, because previous studies done on bulk and thin film MPB PZT have suggested that the tetragonal phase fraction decreases with applied electric field.[19,41-43] Additionally, if a phase transition occurred, the transition would still exhibit some domain texturing in the tetragonal phase, because there is an absence of the 002 at the perpendicular-to-field direction, Fig. 3 (b). This absence of the 002 peak at the perpendicular-to-field direction is typically associated with $90^{\circ}$ domain reorientation. However, the possibility of a phase transition cannot be fully ruled out since the phase identification of this sample is limited by the XRD signal.

In order to quantify the change in the volume fraction of $c$-oriented domains, integrated intensity values for the 002 and 200 reflections were obtained via line-profile analysis. Lineprofile analysis was carried out using two Pearson-VII functions. An example of the peak fit conducted on the 002 and 200 reflection at $20 \mathrm{~V}$ for the parallel-to-field data is shown in Fig. 4. The integrated intensity values for the 002 peak are shown on Fig. 5 (a) and illustrates the 


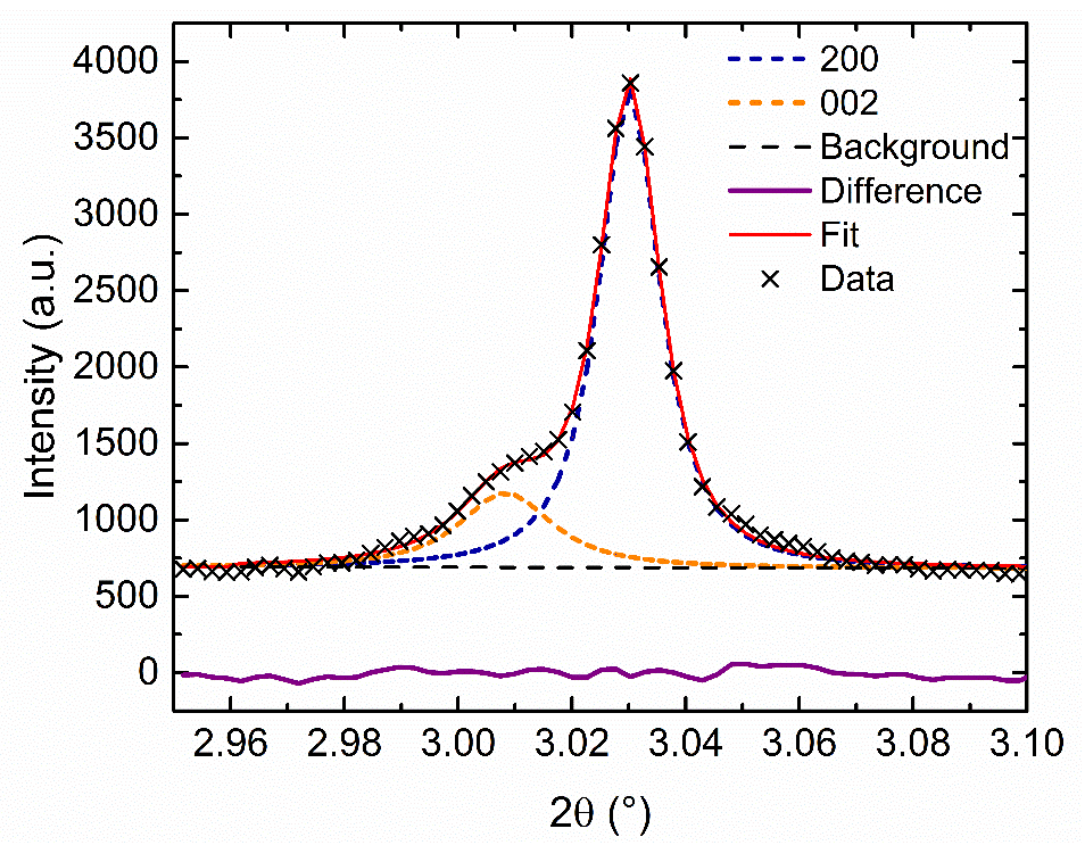

Figure 4 Peak fit of 002 and 200 reflections at 20 V using two Pearson-VII functions.

development of the 002 peak during the experiment. For the $0 \mathrm{~V}$ diffraction pattern only, the integrated intensity value for the 002 peak was assigned to be zero since only the 200 reflection is quantifiable. With increasing voltage, the integrated intensity value for the 002 peak increases as well. From Fig. 5 (a) the integrated intensity values for the 002 peak show that there is a slight hysteresis present in the domain structure after the experiment, so that the integrated intensity values do not fully return to zero after the experiment.

The volume fraction of domains aligned in a given sector $\left(v_{002}\right)$ can be quantified by using Eq. (1),

$$
v_{002}=\frac{\frac{I_{002}}{I_{002}^{\prime}}}{\frac{I_{002}}{I_{002}^{\prime}}+2 \frac{I_{200}}{I_{200}^{\prime}}}
$$

where $I_{002}$ is the integrated intensity of the 002 reflection and $I_{002}^{\prime}$ is the reference intensity from a Powder Diffraction File ${ }^{\mathrm{TM}}$ for the 002 reflection $\left(I_{002}^{\prime}=107, I_{200}^{\prime}=260\right)$ [44]. The ratio of the reference intensities of a Powder Diffraction File ${ }^{\mathrm{TM}}, I_{002}^{\prime}$, and the intensities of the sample, 
$I_{002}$, are used to account for the difference in structure factors for 002 and 200 reflections. From the obtained volume fraction of 002 domains, the fraction of domain reorientation $\left(\eta_{002}\right)$ can be calculated using Eq. (2). In Eq. (2), $v_{002}{ }^{0 \text { field }}$ corresponds to the initial un-poled state of the film. By comparing the induced volume fraction relative to the volume fraction at zero field $\left(v_{002}{ }^{0 \text { field }}\right)$, Eq. (2) would account for an initial domain orientation, if present.

$$
\eta_{002}=v_{002}-v_{002}^{0 \text { field }} \quad \text { Eq. (2) }
$$

The methodology described above has been utilized widely in various polycrystalline ferroelectrics, including both bulk and thin films.[14,20,32,33] Typical $\eta_{002}$ values for electrically poled bulk ferroelectric ceramics can range anywhere from $0.13-0.46$ in the direction parallel-to-the electric field,[40] and 0.02-0.05 for as-processed PZT films on silicon substrates

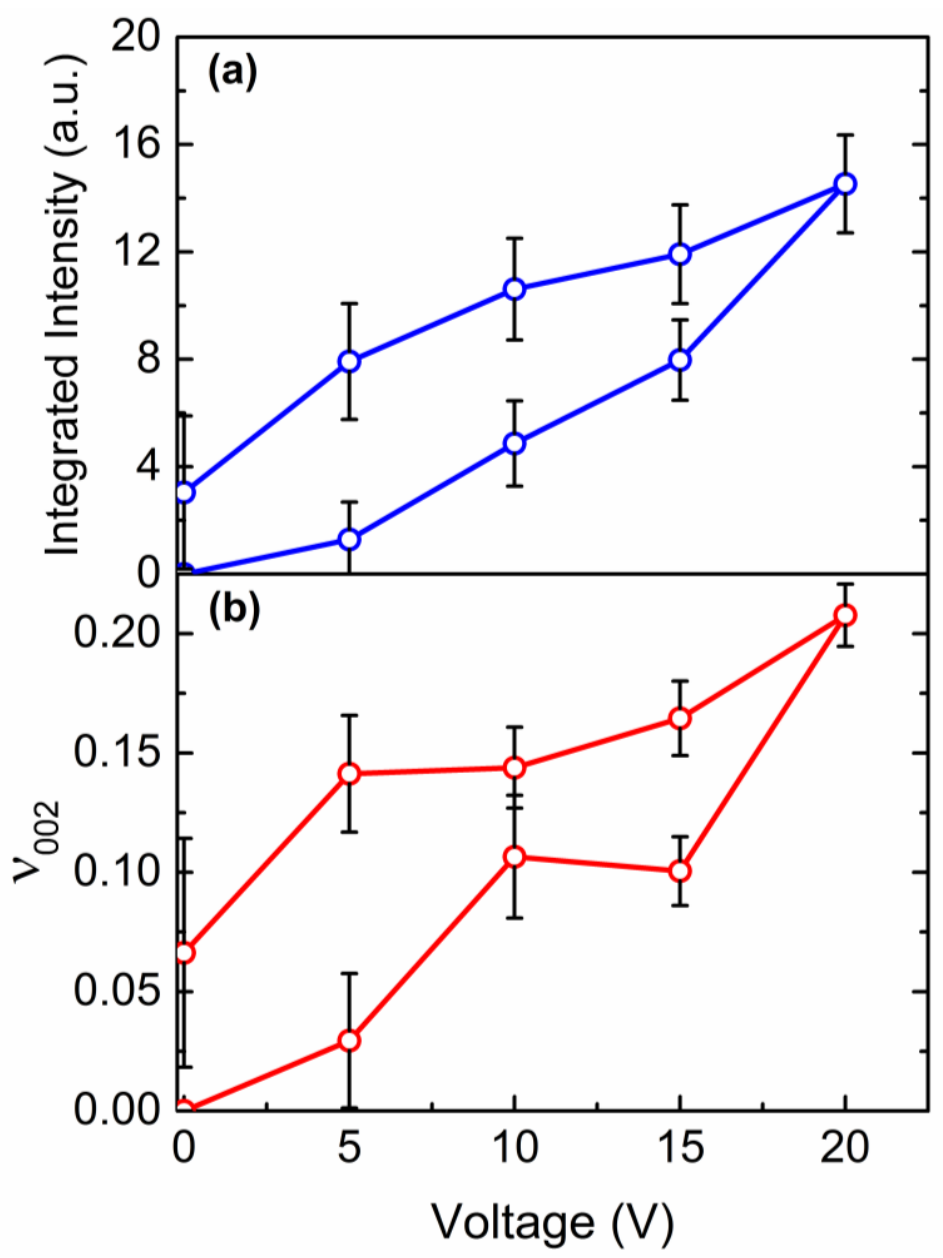

Figure 5 As a function of voltage, (a) integrated intensity values obtained from peak fitting XRD data, and (b) calculated volume fraction of 002 oriented domains. 
that have been electrically poled.[32,33]

Due to the measureable signal of the PZT 002 reflection, as a result of using synchrotron source, Eq. (1) can be used to further quantify the measured diffraction signal. Figure 5 (b) shows the volume fraction of 002 oriented domains as a function of voltage, which was quantified using the XRD data. The maximum volume fraction of 002 oriented domains, seen at $20 \mathrm{~V}$, was $20 \%$. This value of $v_{002}$ is on the order of domain volume fraction changes seen in bulk ferroelectric PZT ceramics and those seen in the study domain by Wallace et al.[32] on released $\{001\}$ tetragonal PZT films. A comparable $v_{002}$ value between the piezoMEMS and bulk is seen as a result of the films initial preferred orientation, $v_{002} 0 \mathrm{~V}$, compared to its induced volume fraction change $v_{002}$. As-processed PZT films on silicon substrates have a tensile in-plane stress, which leads to a high amount of $a$-oriented domains in the in-plane direction of the film as-prepared. This residual stress is known to increase the energy barrier needed for domains to reorient from $a$-domain to $c$-domain,[31] and leads to more $a$-oriented domains in-plane of the film, i.e. higher 200 reflection and lower 002 reflection at $0 \mathrm{~V}$. This allows for a larger amount of $a$-domains to switch to $c$-domains when an electric field is applied, leading to a large value $v_{002}$ that is comparable to bulk ferroelectrics. However, this large $v_{002}$ is a result of substrate removal, which leads to a relief of in-plane tensile stress and lowers the energy barrier for $a$-domain to $c$ domain reorientation, i.e. $90^{\circ}$ domain reorientation. Domain reorientation values for as-processed clamped $\{001\}$ films are typically around 5\%,[32,33] which is a factor of 4 lower than is reported in this study. Therefore, integrating a fully released PZT film into this piezoMEMS device facilitates $90^{\circ}$ domain reorientation due to absence of the underlying substrate. These results suggest that substrate removal will modulate the functional properties of flexural piezoMEMS actuators. 


\section{Conclusions}

High energy XRD confirmed the first instance of $90^{\circ}$ ferroelectric/ferroelastic domain reorientation in a $500 \mathrm{~nm}$ thick PZT layer within a piezoMEMS cantilever during actuation. This device utilized an electrode area that is much smaller than previously reported on similar studies (previous dimensions were, $15 \mathrm{~mm}^{2},[32,33,36]$ ), thus, demonstrating the capability of synchrotron X-rays to probe domain reorientation in small volumes. From the quantified XRD data there was a $20 \%$ increase in the volume fraction of $c$-domains in the parallel-to-field direction at $20 \mathrm{~V}$, which was attributed to the PZT film being released from its underlying substrate. This change in volume fraction is on the order of volume fraction changes seen in other diffraction based ferroelectric bulk studies and work done on $\{001\}$ textured partially released tetragonal PZT films. The large amount of domain reorientation seen in this PZT cantilever provides evidence that the removal of the underlying substrate, which is used to grow the film, facilitates domain reorientation response within films incorporated within piezoMEMS devices. This work also highlights that synchrotron XRD is a useful and powerful characterization tool for interrogating physical mechanisms underpinning the electromechanical response of small length scale ferroelectric materials used in piezoMEMS.

\section{Acknowledgements}

The authors gratefully acknowledge support of this research from the U.S. National Science Foundation (DMR-1410907 and DMR-1409399). The authors acknowledge the use of the Analytical Instrumentation Facility (AIF) at North Carolina State University, which is supported by the State of North Carolina and the National Science Foundation. The authors would like to thank Mr. Jeffrey S. Pulskamp for his design of the microflight actuators and Ms. Mary G. Koebke for fabricating the PCB used for electrical testing. This research used resources 
of the Advanced Photon Source, a U.S. Department of Energy (DOE) Office of Science User

Facility operated for the DOE Office of Science by Argonne National Laboratory under Contract

No. DE-AC02-06CH11357.

\section{References}

[1] P. Muralt, PZT thin films for microsensors and actuators: Where do we stand?, IEEE Trans. Ultrason. Ferroelectr. Freq. Control. 47 (2000) 903-15. doi:10.1109/58.852073.

[2] J.S. Pulskamp, Millimeter-scale MEMS enabled autonomous systems: system feasibility and mobility, 8373 (2012) 83731G. doi:10.1117/12.918670.

[3] S. Trolier-Mckinstry, P. Muralt, Thin film piezoelectrics for MEMS, J. Electroceramics. 12 (2004) 7-17. doi:10.1023/B:JECR.0000033998.72845.51.

[4] P. Muralt, Recent Progress in Materials Issues for Piezoelectric MEMS, J. Am. Ceram. Soc. 91 (2008) 1385-1396. doi:10.1111/j.1551-2916.2008.02421.x.

[5] Q.M. Zhang, H. Wang, N. Kim, L.E. Cross, Direct evaluation of domain-wall and intrinsic contributions to the dielectric and piezoelectric response and their temperature dependence on lead zirconate-titanate ceramics, J. Appl. Phys. 75 (1994) 454-459. doi:10.1063/1.355874.

[6] D.A. Ochoa, G. Esteves, T. Iamsasri, F. Rubio-Marcos, J.F. Fernández, J.E. García, J.L. Jones, Extensive domain wall contribution to strain in a $(\mathrm{K}, \mathrm{Na}) \mathrm{NbO}_{3}$-based lead-free piezoceramics quantified from high energy X-ray diffraction, J. Eur. Ceram. Soc. 36 (2016) 2489-2494. doi:10.1016/j.jeurceramsoc.2016.03.022.

[7] M.C. Ehmke, N.H. Khansur, J.E. Daniels, J.E. Blendell, K.J. Bowman, Resolving structural contributions to the electric-field-induced strain in lead-free (1-x) $\mathrm{Ba}\left(\mathrm{Zr}_{0.2} \mathrm{Ti}_{0.8}\right) \mathrm{O}_{3}$ $\mathrm{x}\left(\mathrm{Ba}_{0.7} \mathrm{Ca}_{0.3}\right) \mathrm{TiO} 3$ piezoceramics, Acta Mater. 66 (2014) 340-348. doi:10.1016/j.actamat.2013.11.021.

[8] S. Trolier-McKinstry, N.B. Gharb, D. Damjanovic, Piezoelectric nonlinearity due to motion of $180^{\circ}$ domain walls in ferroelectric materials at subcoercive fields: A dynamic poling model, Appl. Phys. Lett. 88 (2006). doi:10.1063/1.2203750.

[9] Y. Ehara, S. Yasui, T. Oikawa, T. Shiraishi, N. Oshima, T. Yamada, Y. Imai, O. Sakata, H. Funakubo, Large irreversible non- $180^{\circ}$ domain switching after poling treatment in $\mathrm{Pb}(\mathrm{Zr}, \mathrm{Ti}) \mathrm{O}_{3}$ films, Appl. Phys. Lett. 108 (2016) 212901. doi:10.1063/1.4951672.

[10] M. Nakajima, A. Wada, T. Yamada, Y. Ehara, T. Kobayashi, H. Funakubo, Impact of pulse poling on static and dynamic ferroelastic-domain contributions in tetragonal $\mathrm{Pb}(\mathrm{Ti}, \mathrm{Zr}) \mathrm{O}_{3}$ films determined by in-situ X-ray diffraction analysis, J. Appl. Phys. 116 (2014) 0-7. doi:10.1063/1.4898321.

[11] D. Ghosh, A. Sakata, J. Carter, P.A. Thomas, H. Han, J.C. Nino, J.L. Jones, Domain Wall Displacement is the Origin of Superior Permittivity and Piezoelectricity in $\mathrm{BaTiO}_{3}$ at Intermediate Grain Sizes, Adv. Funct. Mater. 24 (2014) 885-896. doi:10.1002/adfm.201301913.

[12] T. Yamada, J. Yasumoto, D. Ito, O. Sakata, Y. Imai, T. Kiguchi, T. Shiraishi, T. Shimizu, H. Funakubo, M. Yoshino, T. Nagasaki, Negligible substrate clamping effect on piezoelectric response in (111)-epitaxial tetragonal $\mathrm{Pb}(\mathrm{Zr}, \mathrm{Ti}) \mathrm{O}_{3}$ films, J. Appl. Phys. 118 (2015) 072012. doi:10.1063/1.4927810.

[13] J.E. Daniels, T.R. Finlayson, A.J. Studer, M. Hoffman, J.L. Jones, Time-resolved diffraction 
measurements of electric-field-induced strain in tetragonal lead zirconate titanate, J. Appl. Phys. 101 (2007) 094104. doi:10.1063/1.2720255.

[14] J.E. Daniels, W. Jo, J. Rödel, V. Honkimäki, J.L. Jones, Electric-field-induced phase-change behavior in $\left(\mathrm{Bi}_{0.5} \mathrm{Na}_{0.5}\right) \mathrm{TiO}_{3}-\mathrm{BaTiO}_{3}-\left(\mathrm{K}_{0.5} \mathrm{Na}_{0.5}\right) \mathrm{NbO}_{3}$ : A combinatorial investigation, Acta Mater. 58 (2010) 2103-2111. doi:10.1016/j.actamat.2009.11.052.

[15] J.L. Jones, E.B. Slamovich, K.J. Bowman, Domain texture distributions in tetragonal lead zirconate titanate by x-ray and neutron diffraction, J. Appl. Phys. 97 (2005) 034113. doi:10.1063/1.1849821.

[16] T. Key, J.L. Jones, W.F. Shelley, K.J. Bowman, Quantifying Domain Textures in Lead Zirconate Titanate Using 022:202 and 220 Diffraction Peaks, Solid State Phenom. 105 (2005) 379-384. doi:10.4028/www.scientific.net/SSP.105.379.

[17] A. Pramanick, J.E. Daniels, J.L. Jones, Subcoercive Cyclic Electrical Loading of Lead Zirconate Titanate Ceramics II: Time-Resolved X-Ray Diffraction, J. Am. Ceram. Soc. 92 (2009) 23002310. doi:10.1111/j.1551-2916.2009.03219.x.

[18] A. Pramanick, J.L. Jones, Measurement of structural changes in tetragonal PZT ceramics under static and cyclic electric fields using a laboratory X-ray diffractometer., IEEE Trans. Ultrason. Ferroelectr. Freq. Control. 56 (2009) 1546-54. doi:10.1109/TUFFC.2009.1219.

[19] M. Hinterstein, J. Rouquette, J. Haines, P. Papet, M. Knapp, J. Glaum, H. Fuess, Structural Description of the Macroscopic Piezo- and Ferroelectric Properties of Lead Zirconate Titanate, Phys. Rev. Lett. 107 (2011) 077602. doi:10.1103/PhysRevLett.107.077602.

[20] J.L. Jones, M. Hoffman, J.E. Daniels, A.J. Studer, Direct measurement of the domain switching contribution to the dynamic piezoelectric response in ferroelectric ceramics, Appl. Phys. Lett. 89 (2006) 092901. doi:10.1063/1.2338756.

[21] F. Griggio, S. Jesse, A. Kumar, O. Ovchinnikov, H. Kim, T.N. Jackson, D. Damjanovic, S. V. Kalinin, S. Trolier-McKinstry, Substrate Clamping Effects on Irreversible Domain Wall Dynamics in Lead Zirconate Titanate Thin Films, Phys. Rev. Lett. 108 (2012) 157604-5. doi:10.1103/PhysRevLett.108.157604.

[22] M.O. Eatough, D. Dimos, B.A. Tuttle, W.L. Warren, R. Ramesh, A Study of Switching Behavior in $\mathrm{Pb}(\mathrm{Zr}, \mathrm{Ti}) \mathrm{O}_{3}$ Thin Films Using X-Ray Diffraction, MRS Proc. 361 (1994) 111. doi:10.1557/PROC-361-111.

[23] F. Griggio, S. Trolier-McKinstry, Grain size dependence of properties in lead nickel niobate-lead zirconate titanate films, J. Appl. Phys. 107 (2010) 024105. doi:10.1063/1.3284945.

[24] F. Xu, S. Trolier-McKinstry, W. Ren, B. Xu, Z.-L. Xie, K.J. Hemker, Domain wall motion and its contribution to the dielectric and piezoelectric properties of lead zirconate titanate films, J. Appl. Phys. 89 (2001) 1336-1348. doi:10.1063/1.1325005.

[25] N. Bassiri-Gharb, I. Fujii, E. Hong, S. Trolier-McKinstry, D. V. Taylor, D. Damjanovic, Domain wall contributions to the properties of piezoelectric thin films, J. Electroceramics. 19 (2007) 4967. doi:10.1007/s10832-007-9001-1.

[26] D.V. Taylor, D. Damjanovic, Piezoelectric properties of rhombohedral $\mathrm{Pb}(\mathrm{Zr}, \mathrm{Ti}) \mathrm{O}_{3}$ thin films with (100), (111), and "random" crystallographic orientation, Appl. Phys. Lett. 76 (2000) 1615-1617. doi:Doi 10.1063/1.126113.

[27] Y. Bastani, T. Schmitz-Kempen, A. Roelofs, N. Bassiri-Gharb, Critical thickness for extrinsic contributions to the dielectric and piezoelectric response in lead zirconate titanate ultrathin films, J. Appl. Phys. 109 (2011) 014115. doi:10.1063/1.3527970.

[28] T.M. Shaw, S. Trolier-McKinstry, P.C. McIntyre, The Properties of Ferroelectric Films at Small 
Dimensions, Annu. Rev. Mater. Sci. 30 (2000) 263-298. doi:10.1146/annurev.matsci.30.1.263.

[29] G. Catalan, B. Noheda, J. McAneney, L.J. Sinnamon, J.M. Gregg, Strain gradients in epitaxial ferroelectrics, Phys. Rev. B - Condens. Matter Mater. Phys. 72 (2005) 1-4.

doi:10.1103/PhysRevB.72.020102.

[30] B. a. Tuttle, J. a. Voigt, T.J. Garino, D.C. Goodnow, R.W. Schwartz, D.L. Lamppa, T.J. Headley, M.O. Eatough, Chemically prepared $\mathrm{Pb}(\mathrm{Zr}, \mathrm{Ti}) \mathrm{O}_{3}$ thin films: the effects of orientation and stress, ISAF '92 Proc. Eighth IEEE Int. Symp. Appl. Ferroelectr. (1992). doi:10.1109/ISAF.1992.300703.

[31] K.S. Lee, Y.K. Kim, S. Baik, J. Kim, I.S.I.S.I.S. Jung, K. Kim, In situ observation of ferroelectric $90^{\circ}$-domain switching in epitaxial $\mathrm{Pb}(\mathrm{Zr}, \mathrm{Ti}) \mathrm{O}_{3}$ thin films by synchrotron x-ray diffraction, Appl. Phys. Lett. 79 (2001) 2444. doi:10.1063/1.1406981.

[32] M. Wallace, R.L. Johnson-Wilke, G. Esteves, C.M. Fancher, R.H.T. Wilke, J.L. Jones, S. TrolierMcKinstry, In situ measurement of increased ferroelectric/ferroelastic domain wall motion in declamped tetragonal lead zirconate titanate thin films, J. Appl. Phys. 117 (2015) 054103. doi:10.1063/1.4907394.

[33] R.L. Johnson-Wilke, R.H.T. Wilke, M. Wallace, A. Rajashekhar, G. Esteves, Z. Merritt, J.L. Jones, S. Trolier-McKinstry, Ferroelectric/Ferroelastic domain wall motion in dense and porous tetragonal lead zirconate titanate films, IEEE Trans. Ultrason. Ferroelectr. Freq. Control. 62 (2015) 46-55. doi:10.1109/TUFFC.2014.006562.

[34] H. Morioka, K. Saito, T. Kobayashi, S. Yasui, T. Kurosawa, H. Funakubo, In situ Observation of the Fatigue-Free Piezoelectric Microcantilever by Two-Dimensional X-ray Diffraction, Jpn. J. Appl. Phys. 48 (2009) 09KA03. doi:10.1143/JJAP.48.09KA03.

[35] H. Morioka, K. Saito, T. Kobayashi, T. Kurosawa, H. Funakubo, In-Plane Lattice Strain Evaluation in Piezoelectric Microcantilever by Two-Dimensional X-ray Diffraction, Jpn. J. Appl. Phys. 47 (2008) 7537-7540. doi:10.1143/JJAP.47.7537.

[36] G. Esteves, M. Wallace, R. Johnson-Wilke, C.M. Fancher, R.H.T. Wilke, S. Trolier-McKinstry, J.L. Jones, Effect of Mechanical Constraint on Domain Reorientation in Predominantly \{111\}Textured Lead Zirconate Titanate Films, J. Am. Ceram. Soc. 99 (2016) 1802-1807. doi:10.1111/jace.14159.

[37] J.R. Bronson, J.S. Pulskamp, R.G. Polcawich, C.M. Kroninger, E.D. Wetzel, PZT MEMS Actuated Flapping Wings for Insect-Inspired Robotics, in: 2009 IEEE 22nd Int. Conf. Micro Electro Mech. Syst., IEEE, 2009: pp. 1047-1050. doi:10.1109/MEMSYS.2009.4805566.

[38] A.P. Hammersley, S.O. Svensson, M. Hanfland, a. N. Fitch, D. Hausermann, Two-dimensional detector software: From real detector to idealised image or two-theta scan, High Press. Res. 14 (1996) 235-248. doi:10.1080/08957959608201408.

[39] M. Marsilius, T. Granzow, J.L. Jones, Quantitative comparison between the degree of domain orientation and nonlinear properties of a PZT ceramic during electrical and mechanical loading, J. Mater. Res. 26 (2011) 1126-1132. doi:10.1557/jmr.2011.40.

[40] G. Esteves, C.M. Fancher, J.L. Jones, In situ characterization of polycrystalline ferroelectrics using X-ray and neutron diffraction, J. Mater. Res. 30 (2014) 340-356. doi:10.1557/jmr.2014.302.

[41] L. Fan, J. Chen, Y. Ren, Z. Pan, L. Zhang, X. Xing, Unique Piezoelectric Properties of the Monoclinic Phase in $\mathrm{Pb}(\mathrm{Zr} ; \mathrm{Ti}) \mathrm{O}_{3}$ Ceramics : Large Lattice Strain and Negligible Domain Switching, 027601 (2016) 1-5. doi:10.1103/PhysRevLett.116.027601.

[42] K. Schönau, M. Knapp, H. Kungl, M. Hoffmann, H. Fuess, In situ synchrotron diffraction investigation of morphotropic $\mathrm{Pb}\left[\mathrm{Zr}_{1-\mathrm{x}} \mathrm{Ti}_{\mathrm{x}}\right] \mathrm{O}_{3}$ under an applied electric field, Phys. Rev. B. 76 
(2007) 144112. doi:10.1103/PhysRevB.76.144112.

[43] V. Kovacova, N. Vaxelaire, G. Le Rhun, P. Gergaud, T. Schmitz-Kempen, E. Defay, Correlation between electric-field-induced phase transition and piezoelectricity in lead zirconate titanate films, Phys. Rev. B. 90 (2014) 140101. doi:10.1103/PhysRevB.90.140101.

[44] JCPDS Card -International Centre for Diffraction Data ${ }^{\circledR}-\mathrm{Pb}\left(\mathrm{Zr}_{0.52}, \mathrm{Ti}_{0.48}\right) \mathrm{O}_{3}$, (2000) 01-070-4060. 


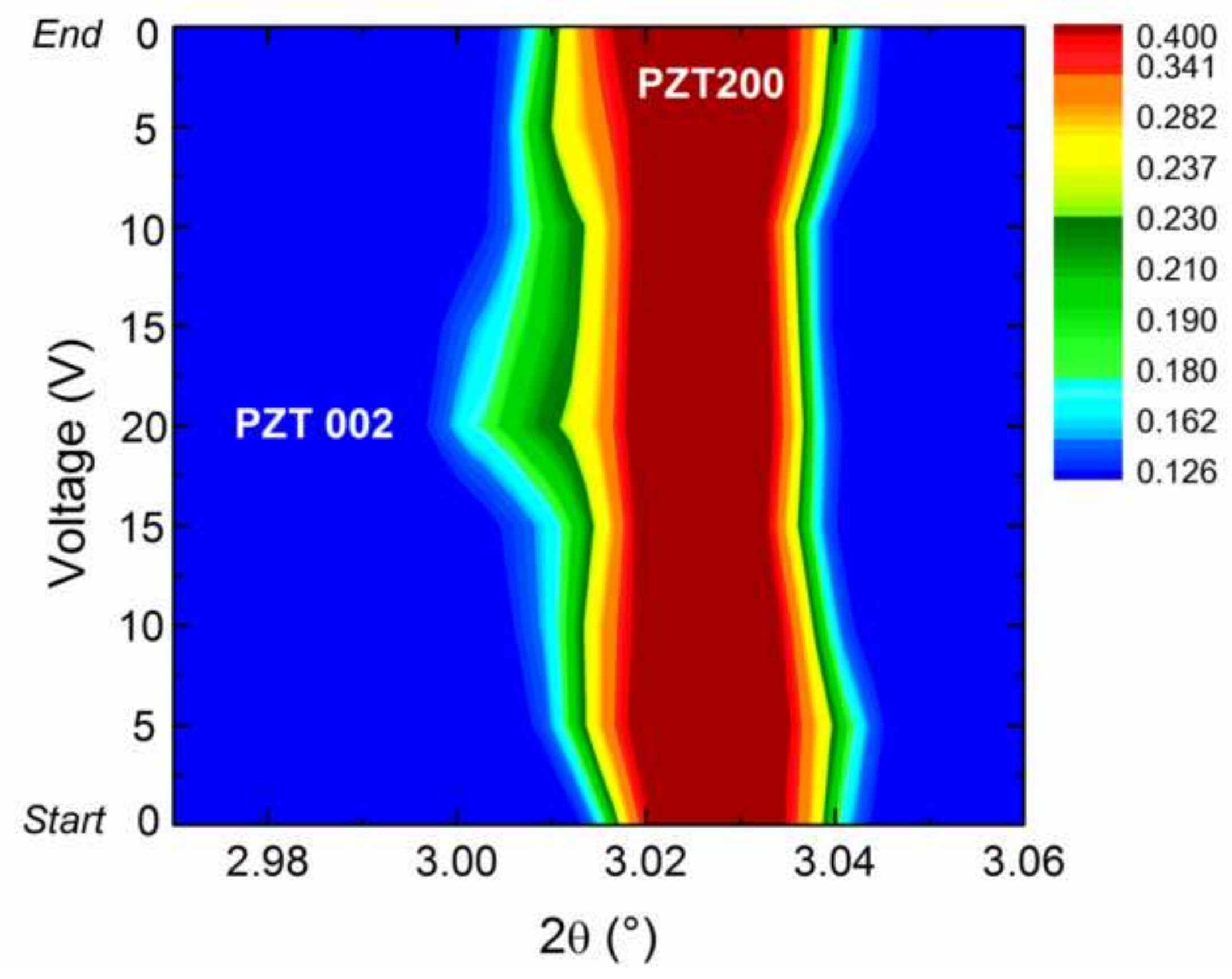

